\section{Broadcasting in India}

Av outline of the policy and plans of the broadcasting organization known as All India Radio for the erection of broadcasting stations in India was given in a recent issue of the Indian Listener. The two main features of the problem of providing a broadcasting service in India are the relatively large area of country to be covered, and the intense atmospheric interference. It is considered to be desirable to provide as quickly as possible some sort of broadcasting service for the whole area of India, and with this object in view five short-wave transmitting equipments have been ordered and will be located at Delhi (two stations), Bombay, Calcutta and Madras. At the same time, five medium-wave stations have been ordered to provide a first-grade broadcasting service for the towns of Lahore, Lucknow, Trichinopoly, Dacca and Madras. These will supplement the existing medium-wave stations at Delhi, Bombay, Calcutta and Peshawar, so that shortly All-India Radio will have in operation five short-wave and nine medium-wave stations, the aerial power ratings of these varying from 0.25 to $10 \mathrm{kw}$. Bearing in mind that, in contrast with the practice in other countries, the short-wave stations have to provide an internal service in India, the operating wave-lengths will probably be between 30 and 50 metres for daytime and between 60 and 90 metres for night working. It is considered unlikely that there will be any interference between these stations working an internal service in India, and European and other short-wave stations operating an international service. The new medium-wave stations will operate on wave-lengths between 200 and 400 metres and will have large frequency separation so as to facilitate the provision of simple cheap receivers.

AT the present time the position with regard to broadcasting receivers in India is unsatisfactory owing to their high cost. It is considered that with the development of transmitting stations now being undertaken, there will shortly be room for three types of receiver. The first is a cheap, popular receiver suitable for local reception from the mediumwave stations. Next comes the "All-India" receiver suitable for receiving all the stations now in contemplation, and thus capable of covering the shortwave band of 30-100 metres as well as the normal medium-wave band of 200-550 metres. The third type of receiver is in the "all-wave" class generally available at the present time in Great Britain, except that it should cover wave-lengths from 13 to 100 metres without a gap. In addition, there is a demand in India for wireless receivers suitable for community reception in villages, and the Research Department of All-India Radio has already developed a special set for this purpose. No external controls are provided on this receiver, which is left tuned to the local station and is operated by a clockwork timeswitch, which turns the receiver on and off at the correct time for the "village hour". The only attention required by these receivers is a visit once every three weeks to change the accumulator battery and re-wind the clock.

\section{Origins of Clerk Maxwell's Electric Ideas}

A воoK entitled "Origins of Clerk Maxwell's Electric Ideas as described in Familiar Letters to William Thomson" has been published by the Cambridge University Press (price 3s. 6d.) These letters cover the period 1854-79 and illustrate clearly the genesis and rapid progress of Clerk Maxwell's ideas as he groped his way towards a structural theory of the electric and magnetic field. Some of the questions he asks Thomson are by no means easy to answer. In his first letter (Feb. 1854) he asks : "Suppose a man to have a popular knowledge of electrical show experiments and a little antipathy to Murphy's Electricity, how ought he to proceed in reading and working so as to get a little insight into the subject which may be of use to him in further reading?" In subsequent letters he continues to ask still more intricate questions, so doubtless Thomson's answers must have been satisfactory. In another letter he says, "I do not know the game laws and patent laws of science . . . but I certainly intend to poach among your electrical images". He fully appreciates Thomson's problem of an electrified spherical bowl. "Your bowl investigations are firstrate. I must find the induction through a round hole in a plate by means of them. Whether would you have me bag the whole thing for my book, or give results and references with an account of the method ?" The letters given in this book were originally printed in Part 5 of vol. 32 of the Proceedings of the Cambridge Philosophical Society. As they will be of interest to many mathematical physicists, the Cambridge Press did well to publish them. Sir Joseph Larmor has edited the book.

\section{Clean Milk and Pasteurization}

Dr. G. Arbour-Stephens, of 61 Walter Road, Swansea, writes, with reference to the article on the nutritive value of pasteurized milk (NATURE, 140, $389 ; 1937)$, that it is not justifiable to compromise a diminution of value in order to prevent the effects of handling by dirty people. Unfortunately, the problem is scarcely as simple as this : in spite of the greatest care and cleanliness in handling the milk, it cannot always be possible to prevent contamination from organisms which may have produced no obvious illness in the worker himself, but yet may be capable of producing illness in other people, or from organisms which may be disseminated during the incubation period of an infectious disease and before the symptoms have become obvious. To prevent otherwise unavoidable outbreaks of infectious diseases among the consumers of milk is the true function of pasteurization : it is certainly not to be considered as allowing the production of dirty milk, which can then be rendered innocuous before consumption. The aim should surely be the production of clean milk from disease-free herds, with pasteurization to obviate the ill-effects of any lapse in technique, which is bound to happen occasionally considering the many stages through which the milk has to pass under modern conditions before it reaches the consumer, or to prevent the accidental entry of virulent organisms. 